\title{
Elaboração e validação de escala diagramática para quantificação da mancha alvo em folhas de acerola
}

\author{
Mercia I.B. Celoto \& Marli F.S. Papa \\ Departamento de Fitossanidade, Engenharia Rural e Solos, Faculdade de Engenharia, Universidade Estadual Paulista- \\ UNESP, 15385-000, Ilha Solteira, SP, Brasil
}

Autor para correspondência: Marli F.S. Papa, e-mail: marlifsp@bio.feis.unesp.br

\begin{abstract}
RESUMO
Os objetivos deste trabalho foram elaborar e validar uma escala diagramática para quantificar a severidade da mancha alvo, causada por Corynespora cassiicola, em folhas de acerola. Foram coletadas folhas apresentando diferentes níveis de severidade da doença no campo. Baseado na lei de estímulo visual de Weber-Fechner, foi elaborada uma escala com os níveis 2, 4, 8, 16, 32 e 48\% de área foliar lesionada. A validação da escala diagramática foi realizada por 10 avaliadores, sem experiência na avaliação de doenças. Estes estimaram a severidade da doença em 50 folhas contendo diferentes níveis de sintomas, mensuradas previamente pelo programa Image Tool. A acurácia e a precisão de cada avaliador foram determinadas por meio de regressão linear simples, entre a severidade real e a estimada. A escala proporcionou bons níveis de acurácia e precisão e os erros absolutos concentraram-se na faixa de 10\%. Além disso, o coeficiente de determinação (precisão das estimativas) com o auxílio da escala apresentou média de $90 \%$ e $78 \%$ sem o auxílio da escala, mesmo sendo inexperientes os avaliadores. A escala proposta foi adequada para as avaliações da severidade da mancha alvo nas folhas de acerola.
\end{abstract}

Palavras-chave: Corynespora cassiicola, Malpighia emarginata, patometria, severidade da doença.

\begin{abstract}
Design and validation of diagrammatic scale for assessment of target spot in Barbados cherry leaf

The objective of this work was to design and validate a diagrammatic scale to assess target spot severity, caused by Corynespora cassiicola, in leaves of Barbados cherry. Leaves with different levels of severity were collected in the field. Following the Weber-Fechner visual stimulus Law, a scale was designed with 2, 4, 8, 16, 32 and 48\% of diseased leaf areas. Validation was carried out by 10 evaluators, without previous practice in assessing diseases, who estimated the severity on 50 leaves with different levels of symptoms measured by the software Image Tool. The accuracy and precision of each rater was determined by simple linear regression between actual and estimated severity. The scale provided good levels of accuracy and precision and the absolute errors were around 10\%. In addition, the coefficient of determination (precision of estimates) presented an average of $90 \%$ with the aid of the scale, and $78 \%$ without the aid of scale. The proposed scale proved to be adequate for severity assessments of target spot of Barbados cherry.
\end{abstract}

Key words: Corynespora cassiicola, Malpighia emarginata, pathometry, disease severity.

A quantificação de doenças é necessária tanto para o estudo de medidas de controle, na determinação da eficiência de um fungicida, na caracterização da resistência varietal, como para a epidemiologia, na construção de curvas de progresso da doença e estimativa dos danos provocados por ela (Amorim, 1995). A quantificação de doenças de plantas pode ser realizada pela incidência ou pela severidade. A severidade de doenças é geralmente estimada visualmente. Para auxiliar o avaliador e minimizar a subjetividade da estimativa, escalas diagramáticas são uma ferramenta útil (Madden et al., 2007). As escalas devem ser de uso fácil, aplicáveis a uma grande faixa de condições, terem resultados reproduzíveis e permitir uma avaliação imediata

Parte da Tese do primeiro autor: Universidade Estadual Paulista. Ilha Solteira SP. 2009.
(Berger, 1980). Vários autores abordam aspectos que devem ser considerados na elaboração de escalas diagramáticas (Horsfall \& Cowling, 1978; Nutter Jr. \& Schultz, 1995; Spósito et al., 2004; Madden et al., 2007).

A mancha alvo, causada pelo fungo Corynespora cassiicola (Berk. \& M.A. Curtis) C.T. Wei, é a principal doença na cultura da acerola (Malpighia emarginata D.C.), na região de Junqueirópolis, SP. Apenas as folhas são afetadas, onde inicialmente observam-se pequenos pontos necróticos circundados por um halo amarelo, que evoluem para manchas maiores com halo clorótico e levam à queda precoce das folhas (Papa, 2005). Apesar da crescente importância da mancha alvo na cultura da acerola brasileira, pouco se sabe sobre esse patossistema. A elaboração e a validação de uma escala diagramática para avaliar a severidade da mancha alvo, constitui ferramenta necessária para compreender a doença sob a influência de 
fatores ambientais e os níveis de resistência do hospedeiro e seu controle. Considerando-se a inexistência de métodos padronizados para quantificar a mancha alvo da acerola, o presente trabalho teve como objetivos desenvolver uma escala diagramática para avaliar a severidade da doença e analisar os níveis de acurácia e precisão das estimativas geradas com sua utilização.

Foram coletadas 100 folhas de acerola (cv. Olivier), em áreas cultivadas no município de Junqueirópolis, SP, com diferentes níveis de severidade da mancha alvo. As folhas foram reproduzidas por fotocópias coloridas e digitalizadas. Com auxílio do programa UTHSCA Image Tool (Versão 3.0, 2002), foram determinadas a área foliar total e a área lesionada de cada folha, obtendo-se a severidade da doença.

Utilizando o valor máximo de severidade da doença constatada nas folhas coletadas, os intervalos da escala foram calculados com o auxílio do programa 2LOG (Tovar-Soto et al., 2002) e os valores obtidos arredondados. Baseandose na lei de Weber-Fechner de acuidade visual (Horsfall \& Cowling, 1978), foi elaborada uma escala diagramática logarítmica com seis níveis de severidade, considerando a forma e distribuição das lesões observadas com maior frequência. Uma vez que as porcentagens de doença a serem representadas na escala foram estabelecidas, fotocópias de folhas de acerola com as lesões e o halo clorótico de mancha alvo coletadas no campo, foram selecionadas para representarem os níveis de severidade determinados.

Para validar a escala diagramática foram selecionadas 50 imagens de folhas digitalizadas com sintomas de mancha alvo em diferentes níveis de severidade. A severidade foi estimada por 10 pessoas, sem experiência na quantificação de doenças, inicialmente, sem o auxílio da escala diagramática, estimando a severidade da doença para cada folha apresentada. Em seguida, estes mesmos avaliadores receberam uma cópia colorida da escala diagramática, estimando novamente a severidade da doença para cada folha de uma segunda sequência de imagens. Posteriormente, estes avaliadores avaliaram novamente uma terceira seqüência de imagens, com a utilização da escala colorida. Não foi realizado treinamento prévio.

A análise da acurácia e precisão das estimativas de severidade de doença de cada avaliador foi realizada por regressão linear simples entre a severidade real, como variável independente, e a severidade estimada pelo avaliador como variável dependente. A acurácia das estimativas de cada avaliador e do conjunto de avaliadores foi determinada pelo teste $\mathrm{t}$ aplicado ao intercepto da regressão linear (a), para verificar a hipótese Ho: $a=0$, e ao coeficiente angular da reta (b), para testar a Ho: b $=1$, ao nível $5 \%$ de probabilidade $(\mathrm{P}=0,05)$. Valores de intercepto significativamente diferentes de 0 (zero) indicam superestimativa $(>0)$ ou subestimativa $(<0)$ da severidade real a níveis baixos de intensidade da doença, enquanto valores de coeficiente angular da reta que desviam significativamente de 1 (um) indicam superestimativa
$(>1)$ ou subestimativa $(<1)$ sistemática da severidade real em todos os níveis de intensidade da doença (Nutter Jr. \& Schultz, 1995).

A precisão das estimativas foi determinada pelo coeficiente de determinação da regressão $\left(R^{2}\right)$ e pela variância dos erros absolutos (severidade estimada menos severidade real) a repetibilidade das estimativas, determinada pela regressão da segunda avaliação em relação à primeira (Nutter Jr. \& Schultz, 1995). As análises de regressão foram efetuadas com o auxílio do programa Microsoft Excel. O valor máximo de severidade da mancha alvo observado nas folhas de acerola coletadas em plantios comerciais foi de $48 \%$, valores maiores levam as folhas à senescência e queda. A escala diagramática para quantificação da severidade dessa doença foi elaborada com seis níveis de severidade, representados pelos valores de 2, 4, 8, 16, 32 e 48\% de área foliar lesionada (Figura 1), considerando a distribuição de sintomas da doença, os níveis de severidade determinados e obedecendo a lei de Weber-Fechner (Horsfall \& Cowling, 1978).

A acurácia das estimativas dos avaliadores, indicativo da proximidade entre valores estimados e reais (Nutter Jr. et al., 1991), foi avaliada pelo coeficiente angular (b) e intercepto $(a)$ da regressão linear entre severidades real e estimada. As avaliações acuradas mostram proximidade entre a estimativa e a realidade. A inclinação da regressão linear entre valores reais e estimados deve ser igual a um, sem desvios sistemáticos, e o intercepto deve ser igual a zero (Leite \& Amorim, 2002). A precisão das estimativas foi averiguada pelo coeficiente de determinação da regressão $\left(\mathrm{R}^{2}\right)$ e a variância dos erros absolutos das estimativas dos avaliadores.

As análises para validação da escala diagramática mostraram que sem o auxílio da escala, $60 \%$ dos avaliadores foram pouco acurados, pois apresentaram valores do intercepto significativamente diferentes de zero para as retas de regressão entre severidade real e estimada, com valores médios de 2,61 (Tabela 1 e Figura 2). Dentre esses avaliadores, apenas os avaliadores $\mathrm{C}$ e $\mathrm{E}$ não superestimaram a severidade, indicando a presença de desvios positivos constantes para a maioria dos avaliadores. O coeficiente angular da reta, na média dos avaliadores, foi de 0,81 e diferiu significativamente de um. Para oito avaliadores $(80 \%)$, os valores do coeficiente angular foram significativamente diferentes de um, indicando a presença de desvios sistemáticos em todos os níveis de intensidade da doença, com tendência à superestimativa (Tabela $1 \mathrm{e}$ Figura 2).

Com a utilização da escala diagramática, a maioria dos avaliadores melhorou os níveis de acurácia, três avaliadores na primeira avaliação e dois avaliadores na segunda avaliação apresentaram valores do intercepto significativamente diferentes de zero, sendo que os demais avaliadores apresentaram desvios positivos constantes. Os valores médios do intercepto na primeira $(1,90)$ e na segunda $(1,77)$ avaliação não diferiram significativamente de zero 


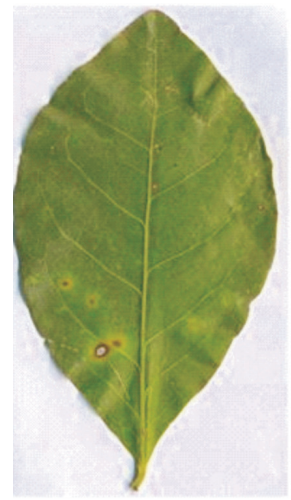

$2 \%$

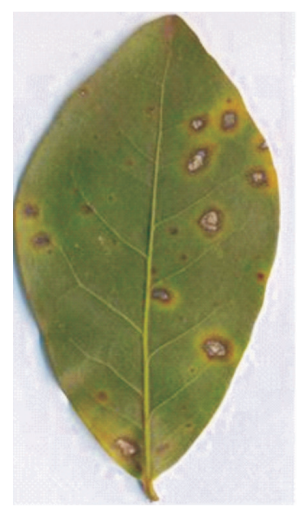

$16 \%$

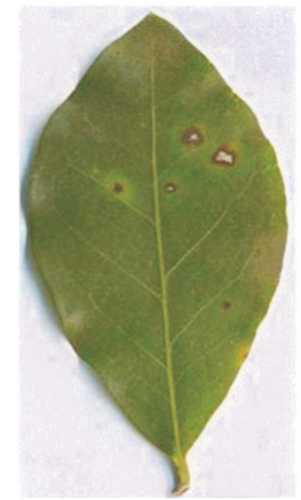

$4 \%$

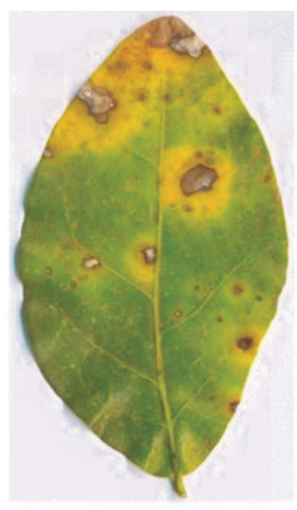

$32 \%$

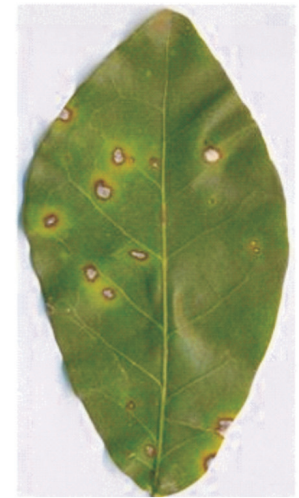

$8 \%$

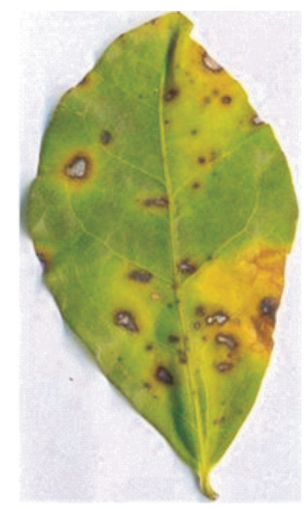

$48 \%$

FIGURA 1 - Escala diagramática para quantificação da mancha alvo em folhas de acerola, com os níveis de $2,4,8,16,32$ e $48 \%$ de severidade da doença.

TABELA 1 - Intercepto (a), coeficiente angular da reta (b) e coeficiente de determinação $\left(\mathrm{R}^{2}\right)$ de equação de regressão linear simples relacionando estimativas visuais da mancha alvo da acerola efetuadas por dez avaliadores, sem e com o auxílio da escala diagramática, à severidade real determinada eletronicamente. Ilha Solteira, SP. 2008

\begin{tabular}{|c|c|c|c|c|c|c|c|c|c|}
\hline \multirow[t]{3}{*}{ Avaliador } & \multicolumn{3}{|c|}{ Sem escala } & \multicolumn{6}{|c|}{ Com escala } \\
\hline & \multirow[b]{2}{*}{$\mathbf{a}$} & \multirow[b]{2}{*}{ b } & \multirow[b]{2}{*}{$\mathbf{R}^{2}$} & \multicolumn{3}{|c|}{$1^{a}$ avaliação } & \multicolumn{3}{|c|}{$2^{a}$ avaliação } \\
\hline & & & & $\mathbf{a}$ & b & $\mathbf{R}^{2}$ & $\mathbf{a}$ & b & $\mathbf{R}^{2}$ \\
\hline $\mathrm{A}$ & 0,02 & $0,68^{*}$ & 0,74 & 1,60 & $0,79^{*}$ & 0,84 & 0,31 & 0,94 & 0,92 \\
\hline $\mathrm{B}$ & 0,18 & $0,83^{*}$ & 0,54 & 0,95 & 0,90 & 0,83 & 1,90 & 1,05 & 0,93 \\
\hline $\mathrm{C}$ & $-1,29$ & $0,70^{*}$ & 0,67 & $3,98^{*}$ & 0,93 & 0,81 & 1,17 & 0,92 & 0,92 \\
\hline $\mathrm{D}$ & $3,49^{*}$ & $0,85^{*}$ & 0,89 & 2,53 & 0,92 & 0,92 & 2,38 & 0,90 & 0,94 \\
\hline $\mathrm{E}$ & $-1,44$ & $0,76^{*}$ & 0,85 & 1,83 & $0,80^{*}$ & 0,87 & 2,17 & $0,88^{*}$ & 0,94 \\
\hline $\mathrm{F}$ & $3,47^{*}$ & $0,79^{*}$ & 0,85 & $-1,66$ & 1,02 & 0,87 & $2,56^{*}$ & $0,86^{*}$ & 0,94 \\
\hline $\mathrm{G}$ & $3,40^{*}$ & 0,97 & 0,88 & $3,02^{*}$ & 0,95 & 0,89 & $3,46^{*}$ & 0,90 & 0,91 \\
\hline $\mathrm{H}$ & $7,24^{*}$ & 0,92 & 0,86 & 0,94 & 0,99 & 0,93 & 1,42 & 0,96 & 0,95 \\
\hline I & $4,81^{*}$ & $0,74^{*}$ & 0,74 & $3,28^{*}$ & 0,93 & 0,87 & 1,41 & 0,96 & 0,94 \\
\hline $\mathrm{J}$ & $6,19^{*}$ & $0,81^{*}$ & 0,80 & 2,57 & 0,92 & 0,82 & 0,97 & 0,95 & 0,90 \\
\hline Média & $2,61^{*}$ & $0,81^{*}$ & 0,78 & 1,90 & 0,92 & 0,87 & 1,77 & 0,93 & 0,93 \\
\hline
\end{tabular}

Asterisco representa situações onde a hipótese de nulidade $(\mathrm{a}=0$ ou $\mathrm{b}=1)$ foi rejeitada pelo teste $\mathrm{t}, \mathrm{p}=0,05$. 
(Tabela 1), indicando a redução dos erros verificados sem o auxílio da escala. O uso da escala resultou em coeficientes angulares similares a um para $80 \%$ dos avaliadores nas duas avaliações, indicando a redução significativa dos erros sistemáticos e melhoria da acurácia das estimativas da reta (Tabela 1 e Figura 2).

A precisão, definida como a exatidão de uma operação onde há rigor ou refinamento na medida (Bergamin Filho \& Amorim, 1996), pode ser avaliada pelo coeficiente de determinação da regressão, que deve ser próximo de $100 \%$ e pela variação dos erros absolutos (diferenças entre severidade estimada e real) (Leite \& Amorim, 2002). Sem o auxílio da escala, o coeficiente de determinação variou de 0,54 a 0,89 . Enquanto que, com o auxílio da escala, o coeficiente de determinação variou de 0,81 a 0,93 na primeira e de 0,90 a 0,95 na segunda avaliação (Tabela 1). Portanto, com o auxílio da escala diagramática os avaliadores tornaram-se mais precisos, mesmo sendo inexperientes. Essa melhoria tem sido verificada com a utilização de outras escalas diagramáticas (Michereff et al., 2006; Angelotti et al., 2008), o que demonstra a importância dessa ferramenta em estudos de quantificação de doenças.

A precisão também foi medida pelos erros absolutos, que são as diferenças entre as severidades estimada e real. De acordo com Nutter Jr. (1989), para que um avaliador possa ser considerado excelente, o erro de suas estimativas deve estar dentro de um intervalo de $\pm 5 \%$ do valor real, e bom quando não ultrapassa a $\pm 10 \%$. Nesse sentido, os avaliadores foram considerados bons na avaliação da severidade da mancha alvo da acerola, pois a média dos erros absolutos permaneceu no intervalo de +10 a $-10 \%$ quando os avaliadores utilizaram a escala diagramática (Figura 3). Segundo Stonehouse (1994), a presença de certo nível de erro absoluto nas mensurações pode ser compensada pela rapidez e padronização quando se utiliza a escala diagramática.

Verificou-se boa repetibilidade das estimativas da severidade da mancha alvo da folha da acerola com o auxílio da escala diagramática, pois a quantidade média de variação na primeira avaliação explicada pela segunda avaliação foi de $88 \%$ (Tabela 2). Os valores médios do intercepto e do coeficiente angular da reta não diferiram significativamente de zero e 1, respectivamente. Entre as duas avaliações, somente para dois avaliadores (B e F) os valores do intercepto foram significativamente diferentes de zero e para dois avaliadores $(\mathrm{C} \mathrm{e} \mathrm{F})$ os valores do coeficiente angular da reta foram significativamente diferentes de 1 , indicando a elevada precisão das estimativas para a maioria dos avaliadores. A quantificação da mancha alvo nas folhas de acerola com o uso da escala diagramática proposta proporcionou mais rapidez e facilidade na avaliação, boas acurácia e precisão. A escala será útil em trabalhos de seleção de cultivares resistentes ao patógeno, estudos epidemiológicos e de desenvolvimento de estratégias de controle da doença.
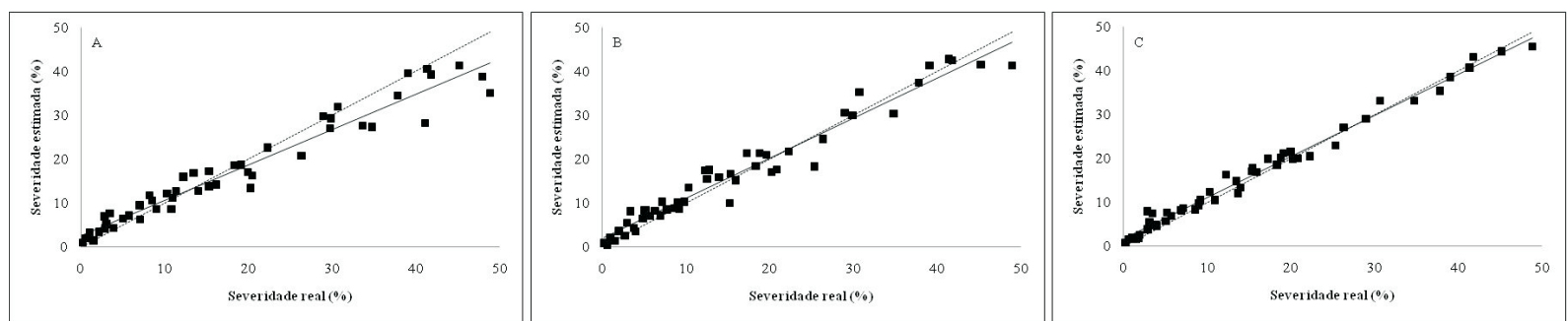

FIGURA 2 - Severidade estimada sem o auxílio (A) e com o auxílio da escala diagramática para mancha alvo da acerola na primeira (B) e segunda (C) avaliação. A linha cheia representa a regressão linear entre a severidade real e a estimada. A linha tracejada representa o ajuste ideal em que a severidade real é igual à estimada. Ilha Solteira, SP. 2008.
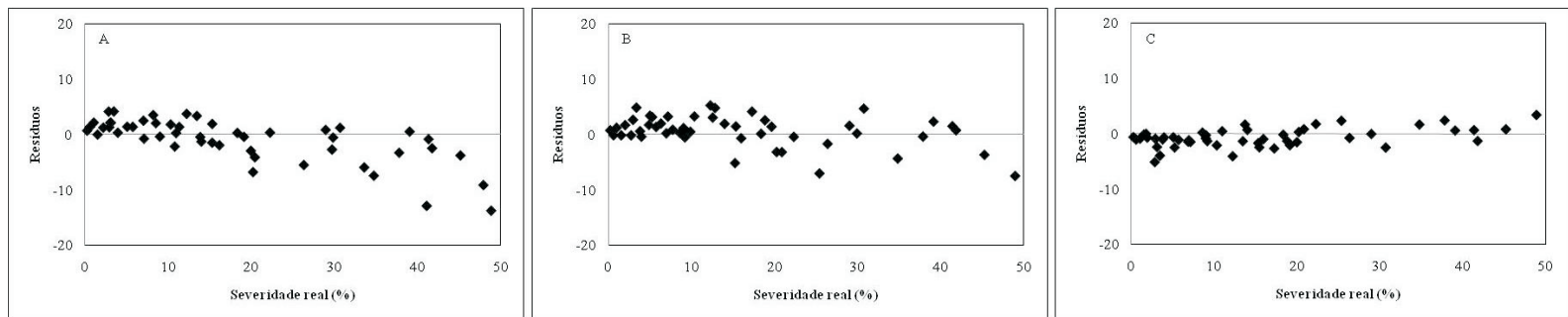

FIGURA 3 - Resíduos (severidade estimada - severidade real) das estimativas da mancha alvo da acerola sem o auxílio da escala diagramática (A) e com o auxílio da escala diagramática na primeira (B) e segunda (C) avaliação. Ilha Solteira, SP. 2008. 
TABELA 2 - Intercepto (a), coeficiente angular da reta (b) e coeficiente de determinação $\left(\mathrm{R}^{2}\right)$ de equação de regressão linear simples relacionando a segunda à primeira estimativa da mancha alvo da acerola efetuadas por dez avaliadores, sem e com o auxílio da escala diagramática, à severidade real determinada eletronicamente. Ilha Solteira, SP. 2008

\begin{tabular}{crcc}
\hline \hline Avaliador & $\mathbf{a}$ & $\mathbf{b}$ & $\mathbf{R}^{\mathbf{2}}$ \\
\hline A & $-0,41$ & 1,03 & 0,85 \\
B & $3,44^{*}$ & 0,99 & 0,84 \\
C & $-0,19$ & $0,88^{*}$ & 0,85 \\
D & 0,84 & 0,92 & 0,91 \\
E & 1,48 & 0,98 & 0,91 \\
F & $3,95^{*}$ & $0,85^{*}$ & 0,92 \\
G & 1,20 & 0,91 & 0,92 \\
H & 1,02 & 0,92 & 0,94 \\
I & 0,10 & 0,95 & 0,89 \\
J & 1,02 & 0,90 & 0,80 \\
\hline Média & 1,24 & 0,93 & 0,88 \\
\hline
\end{tabular}

Asterisco representa situações onde a hipótese de nulidade $(\mathrm{a}=0$ ou $b=1$ ) foi rejeitada pelo teste $t, p=0,05$.

\section{AGRADECIMENTOS}

Os autores manifestam agradecimento ao Prof. Dr. Sami Jorge Michereff, pelo auxílio na elaboração da escala proposta e pela revisão do artigo, ao Prof. Dr. João Antonio da Costa Andrade, pela consultoria estatística e à Fundação de Amparo à Pesquisa do Estado de São Paulo - FAPESP pelo financiamento do projeto (Processo no 07/07386-0).

\section{REFERÊNCIAS BIBLIOGRÁFICAS}

Amorim L (1995) Avaliação de doenças. In: Bergamin Filho A, Kimati H, Amorim L (Eds.) Manual de Fitopatologia. Vol. 1. Princípios e Conceitos. $3^{\text {a }}$. Ed. São Paulo SP. Ceres. pp. 647-671.

Angelotti F, Scapin CR, Tessmann DJ, Vida JB, Oliveira RR, Canteri MG (2008) Diagrammatic scale for assessment of grapevine rust. Tropical Plant Pathology 33:439-443.

Bergamin Filho A, Amorim L (1996) Doenças de plantas tropicais: epidemiologia e controle econômico. São Paulo SP. Ceres.
Berger RD (1980) Measuring disease intensity. In: Teng PS, Krupa SV (Eds.) Crop loss assessment. Saint Paul MN. University of Minnesota. pp. 28-31.

Horsfall JG, Cowling EB (1978) Pathometry: the measurement of plant disease. In: Horsfall JG, Cowling EB (Eds.) Plant disease an advanced treatise. Vol. 2. How disease develops in populations. New York NY. Academic Press. pp. 119-136.

Leite RMVBC, Amorim L (2002) Elaboração e validação de escala diagramática para mancha de Alternaria em girassol. Summa Phytopathologica 28:14-19.

Madden LV, Hughes G, Bosch F (2007) The study of plant disease epidemics. Saint Paul MN. APS Press.

Michereff SJ, Andrade DEGT, Noronha MA (2006) Elaboração e validação de escala diagramática para avaliação da severidade do carvão da folha do caupi. Summa Phytopathologica 32:51-56.

Nutter Junior FW (1989) Disease Pro: a computer program for evaluating and improving a person ability to assess disease proportion. Phytopathology 29:1135.

Nutter Junior FW, Schultz PM (1995) Improving the accuracy and precision of disease assessment: Selection of methods and use of computer-aided training programs. Canadian Journal of Plant Pathology 17:174-178.

Nutter Junior FW, Teng PS, Shokes FM (1991) Disease assessment terms and concepts. Plant Disease 75:1187-1188.

Papa MFS (2005) Doenças da acerola (Malpighia emarginata). In: Kimati H, Amorim L, Rezende JAM, Bergamin Filho A, Camargo LEA (Eds.) Manual de Fitopatologia. Vol. 2. Doenças das Plantas Cultivadas. $4^{\text {a }}$. Ed. São Paulo SP. Ceres. pp.15-18.

Spósito MB, Amorim L, Belasque Junior J, Bassanezi RB, Aquino R (2004) Elaboração e validação de escala diagramática para avaliação da severidade da mancha preta em frutos cítricos. Fitopatologia Brasileira 29:81-85.

Stonehouse J (1994) An assessment of Andean bean disease using visual keys. Plant Pathology 43:519-527.

Tovar-Soto A, Hernandez-Martínez M, Cristóbal-Alejo J, RomeroHijo R, Mora-Aguilera G (2002) Escala logarítimica diagramática de severidad de la mancha negra (Colletotrichum gloeosporioides Penz.) en chirimoyo (Annona cherimola Mill). Revista Mexicana de Fitopatología 20:103-109.

UTHSCA Image Tool Version 3.0 Final. S. Brent Dove. dove@ uthscsa.edu Dental Diagnostic Science, Last revised: 22 February 2002 . 